\title{
Archives
}

\section{La normalisation et la gestion intégrée des documents (GID) : quelle relation? Réflexion sur les normes ISO 30300, ISO 30301, ISO 14641 et leur apport à l'implantation des systèmes de GID}

\section{Siham Alaoui}

Volume 47, numéro 1, 2017

URI : https://id.erudit.org/iderudit/1041824ar

DOI : https://doi.org/10.7202/1041824ar

Aller au sommaire du numéro

Éditeur(s)

Association des archivistes du Québec (AAQ)

ISSN

0044-9423 (imprimé)

2369-9256 (numérique)

Découvrir la revue

Citer cet article

Alaoui, S. (2017). La normalisation et la gestion intégrée des documents (GID) quelle relation? Réflexion sur les normes ISO 30300, ISO 30301, ISO 14641 et leur apport à l'implantation des systèmes de GID. Archives, 47(1), 7-30. https://doi.org/10.7202/1041824ar
Résumé de l'article

Les organisations se servent des documents produits et reçus quotidiennement pour la réalisation effective de leurs activités. Avec la production documentaire en croissance exponentielle, ces organisations tendent à implanter des systèmes de gestion documentaire dans l'objectif de pallier les différents problèmes liés à la gestion, au repérage et à la conservation des documents. Au Québec, les organisations ont commencé à implanter des systèmes de gestion intégrée des documents (GID), visant à gérer les documents dans la diversité de leurs supports et tout au long de leur cycle de vie. La nature complexe de ces systèmes remet en question leur implantation réussie, d'où la nécessité de se doter de lignes directrices ou d'un cadre normatif servant à piloter ces projets. Les normes de l'Organisation internationale de normalisation (ISO), soit ISO 30300 et ISO 30301 pour les systèmes de gestion des documents d'activité, ainsi que l'ISO 14641 pour l'archivage légal et probatoire des documents constituent un cadre normatif pertinent pour l'implantation réussie et l'utilisation effective des systèmes de GID dans les organisations. Cet article présente une réflexion sur l'apport de ces normes à l'implantation de ces systèmes, en partant de la perspective québécoise en archivistique. 


\section{ÉTUDE}

\section{La normalisation et la gestion intégrée des documents (GID): quelle relation? Réflexion sur les normes ISO 30300, ISO 30301, ISO 14641 et leur apport à l'implantation des systèmes de GID}

\section{SIHAM ALAOUI}

Responsable du service de documentation et des archives, Institut supérieur de traduction à Rabat, Maroc

\section{INTRODUCTION}

Dans un environnement d'affaires marqué par le changement et la complexité, les organisations produisent, dans l'exercice de leurs activités quotidiennes, une large variété de documents. Ces documents sont utilisés à des fins de prise de décision et de conduite des activités journalières. Ils constituent également un patrimoine organisationnel dont la valeur est incontournable dans le témoignage de l'existence des organisations et de leurs réalisations à travers le temps. 
Désirant se doter d'une bonne gouvernance d'information, se conformer aux exigences réglementaires en matière de gestion documentaire et valoriser leur mémoire organisationnelle, les organisations tendent à améliorer leurs pratiques de gestion des documents par l'adoption de nouveaux outils et la mise en place de nouveaux systèmes de gestion documentaire plus performants (Beaupré, 2010-2011; Chevrier, 2010-2011). C'est dans cette perspective que les organisations québécoises ont commencé à utiliser une nouvelle pratique archivistique, dite de gestion intégrée des documents (GID). Comme son nom l'indique, elle consiste à gérer les documents dans la diversité de leurs supports par le même système informatique. Les apports des systèmes de GID sont multiples pour les organisations qui les ont utilisés. Citons, entre autres, la bonne gestion des documents et leur repérage amélioré (Bourhis, 2009-2010; Couture, 2010-2011) afin d'optimiser le processus de la prise de décision et d'améliorer les performances organisationnelles. Néanmoins, la réussite des projets d'implantation des systèmes de GID n'est pas acquise: certaines organisations éprouvent des difficultés quant à la réalisation de ces projets complexes qui requièrent d'énormes efforts de la part des intervenants. Ces organisations ont besoin d'outils de pilotage afin de contrôler ces projets et de veiller à leur conduite dans les meilleures conditions. C'est le rôle rempli par les normes nationales et internationales.

L'apport de la normalisation à l'implantation des systèmes de GID a fait l'objet de peu d'écrits scientifiques et professionnels, et ce, en raison du caractère novateur de la GID dans plusieurs pays. C'est ainsi que nous suggérons, dans cet article, de mettre l'accent sur les normes les plus connues en archivistique, soit la série des normes ISO 30300 et ISO 30301 (2011) concernant la mise en place d'un système de gestion des documents d'activité (SGDA), ainsi que la norme ISO 14641 (2012) relative à l'archivage pérenne et probatoire des documents numériques. Plus particulièrement, nous nous attardons à l'apport de chacune de ces normes à la mise en place et à la performance des systèmes de GID, à la lumière des exigences techniques, archivistiques et légales en vigueur.

Nous présenterons, à travers les lignes subséquentes, la GID en tant que pratique archivistique novatrice et la positionnons par rapport à l'archivistique et à la gestion des documents d'activité (c'est-à-dire gestion des archives courantes et intermédiaires). Nous décrirons ensuite 
les fonctionnalités des systèmes de GID. Puis, nous nous pencherons sur la normalisation et son apport aux pratiques de gestion documentaire. Pour ce faire, nous aborderons les normes mentionnées plus haut et nous suggérerons une réflexion sur leur contribution à l'uniformisation des pratiques de la GID.

Une consultation d'un ensemble de ressources documentaires a été nécessaire afin de repérer les articles scientifiques et professionnels traitant du sujet en question: les bases de données bibliographiques (LISA : Library and Information Science Abstracts, EBSCO et Francis), les ensembles de périodiques (Cairn, Érudit et Emerald journals), les catalogues (Atrium de I'Université de Montréal et celui de la Bibliothèque nationale de France $(\mathrm{BnF})$ ), les revues scientifiques (Information management journal, Documentaliste-Sciences de l'information et Records management journal), les revues professionnelles (La Gazette des archives et Archives), ainsi que le système universitaire de documentation (SUDOC). Les mots-clés utilisés étaient en français et en anglais: gestion intégrée des documents, gestion des documents d'activité, gestion des archives courantes et intermédiaires, gestion électronique des documents, documents d'activité, documents d'archives, normalisation, normes, ISO 30300, et ISO 30301, ISO 14641, systèmes de gestion des documents, records, electronic records management, document and archive management, information systems implementation, standards et standardisation.

\section{LA GESTION INTÉGRÉE DES DOCUMENTS (GID): VERS UNE NOUVELLE PRATIQUE ARCHIVISTIQUE}

La gestion intégrée des documents (GID) est une pratique archivistique moderne qui assure la gestion du cycle de vie complet des documents, peu importe leur support, dès leur création jusqu'à leur destruction ou leur conservation permanente. D'un point de vue technique, cette gestion est assurée par le même processus et utilise les mêmes outils puis est intégrée à un seul système informatique (Roberge, 2009 et 2016 ; Université du Québec à Montréal, s.d.). D'un point de vue opérationnel, un système de GID est:

Un système pérenne de gestion dont les composantes sont intégrées afin de donner, aux personnes accréditées, accès à toute l'information pertinente portée par un support qui 
documente les activités d'une organisation. (Roberge, 2009, chapitre 6.1)

Roberge (2009) ajoute que le concept de GID est plus global que celui de la GED, c'est-à-dire gestion électronique des documents. Cette dernière se définit comme étant la gestion du cycle complet des documents numériques, et ce, depuis leur création jusqu'à leur conservation ou leur destruction (LeSaux, 1992). La GID s'inscrit ainsi dans une vision holistique qui considère la notion du document dans l'une de ses acceptations les plus larges en archivistique, selon des critères tels que la diversité des supports documentaires (par exemple, papier, microfilm, audiovisuel, cartographique, etc.) et les étapes du cycle de vie (records ou documents d'activité, ou encore les archives courantes et intermédiaires, ainsi que les archives définitives ou historiques).

\subsection{La GID: un bref aperçu historique}

La GID a vu le jour au Québec, après I'adoption de la Loi concernant le cadre juridique des technologies de l'information' (RLRQ, chapitre C-1.1) (Roberge, 2009, chapitre 6). Cette dernière fournit des règles à suivre afin d'assurer une gestion optimisée des documents « faisant appel aux technologies de l'information» (Loi concernant le cadre juridique des technologies de l'information, RLRQ, chapitre C-1.1, chapitre 1) et produits par les organisations québécoises dans l'exercice de leurs activités. Plus précisément, elle détaille les mesures pour le maintien de I'authenticité2, de la fiabilitée ${ }^{3}$, de l'intégrité ${ }^{4}$, de l'exploitabilité ${ }^{5}$ et de la sécurité de ces documents. II en va de même pour les systèmes de gestion des documents numériques, lesquels doivent être aussi performants que possible afin de garantir les exigences précédemment mentionnées. Ces dispositions visent à garantir la valeur légale des documents permettant aux institutions concernées de se défendre en cas de litige.

Bibliothèque et Archives nationales du Québec (BAnQ) et le Centre des services partagés du Québec (CSPQ) étaient les premières organisations à avoir adopté un modèle conceptuel portant sur la gestion des documents d'activité en formats papier et numérique sur la base de la GID (Roberge, 2009, chapitre 6). L'élaboration et I'application de ce modèle étaient réalisées dans une optique de minimiser l'intensité des problèmes liés à la gestion des documents dans la diversité de leurs supports, notamment avec 
I'avènement du mythe "zéro papier » et l'accroissement du mouvement de la dématérialisation des documents. Nombreuses sont les organisations ayant élaboré des politiques de gestion documentaire adaptées à leur réalité organisationnelle, comme la Stratégie canadienne de numérisation du patrimoine documentaire, adoptée par Bibliothèque et Archives Canada ${ }^{6}$, le Cadre de référence gouvernemental en gestion intégrée des documents ${ }^{7}$, élaboré en 2004 par Bibliothèque et Archives nationales du Québec, en collaboration avec Yves Marcoux, et autres, ainsi que le module de formation proposé par le Conseil international des archives, intitulé UNESCO digital preservation initiatives module ${ }^{8}$ et rédigé en mai 2016. II importe également de mettre l'accent sur des projets connus à l'échelle internationale, et ayant comme finalité la gestion des documents numériques, soit InterPARES (International research on permanent authentic records in electronic systems), ayant pour but la conservation pérenne des documents numériques tout en s'assurant de leur authenticité, ainsi que le projet Hector (Hybrid electronic curation, transformation and organisation of records) lancé en Belgique pour la gestion des documents en formats papier et numérique par un seul système hybride (Hector project, 2016). Par conséquent, force est d'admettre que les instances nationales et internationales sont de plus en plus conscientes des risques découlant de la gestion des documents numériques et de leur préservation pérenne, entre autres: I'impossibilité de tout stocker dans les serveurs informatiques, I'instabilité des supports informatiques (nécessité continue de migration des supports, compte tenu de leur caractère volatile), I'intensité des exigences juridiques obligeant les organisations à disposer les documents servant à leur défense, la multiplicité des risques encourus avec le numérique à l'égard de la sécurité de l'information et l'accentuation des risques liés à la perte de la mémoire organisationnelle.

Les documents papier, quant à eux, continuent d'être produits dans les organisations d'une façon décentralisée dans les différents services (Mas, 2007), ce qui entrave leur bonne gestion et leur repérage efficace. Dans l'impossibilité de tout stocker dans les entrepôts et dans l'obligation d'opter pour des mesures de sécurité visant à préserver les documents essentiels à la conduite des activités quotidiennes des organisations, il s'avère crucial d'opter pour des mesures rationnelles visant à assurer la gestion sécurisée de ces documents au moindre coût.

La GID semble ainsi être la pratique propice pour assurer la gestion des documents en formats papier et numérique, tout en palliant les problèmes 
identifiés plus haut. Par conséquent, cela contribuera à l'amélioration des performances organisationnelles et à la maximisation de l'usage des documents à des fins de prise de décision. Concrètement, elle vise à remplir un ensemble d'objectifs (Beaupré, 2010-2011; Bourhis, 2009-2010 ; Margulis, 2009-2010; Roberge, 2009 et 2016). Elle assure la gestion efficiente, intégrée et corporative des documents en tant que ressources informationnelles, et ce, peu importe le support. Elle favorise un repérage efficace de l'information, grâce à l'utilisation d'un plan de classification uniforme (pour l'ensemble des documents, dans la diversité de leurs supports) et, dans la mesure du possible, d'un thésaurus avec une liste des mots-clés et des noms des personnes contrôlés. En outre, elle la GID aide à la réduction des problèmes liés au stockage de l'information, par I'utilisation d'un calendrier de conservation énumérant et définissant les règles de conservation pour chaque type de document. Aussi, elle minimise les risques liés à la perte de l'information essentielle (vitale), et ce, grâce à l'attribution des règles d'accès et l'identification obligatoire des utilisateurs des systèmes de GID afin de protéger les documents contre les modifications non autorisées. Enfin, elle renforce la conformité aux exigences légales et réglementaires en matière de gestion des documents, entre autres: la Loi sur les $\operatorname{archives}^{9}$ (RLRQ, chapitre A-21.1), la Loi concernant le cadre juridique des technologies de l'information (RLRQ, chapitre $\mathrm{C}-1.1$ ) et la Loi sur l'accès aux documents des organismes publics et sur la protection des renseignements personnels ${ }^{10}$ (RLRQ, chapitre A-2.1).

\subsection{La GID, I'archivistique et la gestion des documents d'activité: quelle relation?}

Relevant de I'archivistique moderne, la GID est une pratique qui s'inscrit dans un mouvement où le numérique occupe une place considérable. Elle vise à renforcer les fondements de l'archivistique en fournissant des mesures permettant aux documents de mieux satisfaire les quatre fameuses exigences archivistiques, soit l'authenticité, l'intégrité, la fiabilité et l'exploitabilité. Elle permet également aux documents de remplir leurs deux principales fonctions, à savoir la fonction de preuve, qui renvoie à l'utilité des documents pour la prise de décision et la défense dans les cas de litiges, et la fonction d'information, selon laquelle ces documents permettent de renseigner sur les activités et les réalisations des organisations à travers le temps. Contrairement 
à la gestion des documents d'activité qui ne concerne - comme son nom l'indique - que la gestion des archives courantes et intermédiaires, la GID porte sur l'ensemble du cycle de vie des documents papier et numériques, depuis leur création jusqu'à leur élimination ou leur conservation permanente.

\subsection{Les systèmes de GID: fonctionnalités et composantes}

\subsubsection{Fonctionnalités des systèmes de GID}

Les systèmes de GID sont des systèmes hybrides offrant un ensemble de fonctionnalités propres aux systèmes de gestion documentaire, qui s'appliquent à tous les documents, peu importe le support ou le stade du cycle de vie. Parmi ces fonctionnalités, on retient (Roberge, 2009, chapitre 8): I'identification et l'enregistrement des documents entrant dans le système à l'aide d'un numéro basé sur la classe (c'est-à-dire la catégorie) à laquelle le document appartient dans le plan de classification, leur description (par exemple, le titre, le nom du producteur et l'activité traitée), leur classement à l'aide d'une cote classificatoire conformément au plan de classification établi, leur indexation (en vocabulaire libre ou contrôlé) pour un meilleur repérage et enfin, leur consultation qui tient en compte les règles d'accès définies pour maintenir la confidentialité de ces documents et leur protection contre les modifications non autorisées. Les systèmes de GID assurent la gestion de l'ensemble du cycle de vie des documents (c'est-à-dire depuis leur création jusqu'à leur élimination ou leur conservation permanente). Le sort final des documents (conservation permanente ou élimination) est défini conformément aux règles de conservation établies pour les types des documents. Ceux qui sont destinés à être conservés sont archivés dans le système, en se souciant de leur pérennité et de leur intégrité.

\subsubsection{Composantes des systèmes de GID}

Comme tous les systèmes d'information (SI), les systèmes de GID comprennent un ensemble d'éléments en interaction en vue de réaliser une ou plusieurs tâches précises (O'Brien, 2003). Dans le cas des systèmes de GID, la tâche est I'harmonisation et l'optimisation de l'ensemble des opérations relevant de la gestion documentaire, à savoir les fonctionnalités du système de GID précédemment mentionnées. 
La figure 1 ci-dessous révèle que le système de GID reçoit de I'information (documents en formats papier et numérique) produite ou reçue par l'organisation, mais qui n'est pas organisée (entité: intrants). Ces informations sont destinées à être gérées en vue de fournir des livrables utiles (documents) pour la prise de décision (extrants). Pour ce faire, ces documents sont enregistrés dans le système à l'aide d'un identifiant. Ils sont ensuite décrits par des éléments de base, entre autres: le titre, le nom du producteur, l'activité décrite, la date et le lieu de production. Ces documents sont classés dans la catégorie correspondante du plan de classification représentant les grandes fonctions de l'organisation et les activités qui en découlent, et ce, dans une logique hiérarchique tenant compte des liens organiques entre les documents (appartenance au dossier du même producteur ou au dossier regroupant les pièces traitant de la même affaire, etc.). Puis, les documents sont indexés par des termes contrôlés afin de faciliter la recherche documentaire. On leur attribue également des règles $d$ 'accès afin d'assurer le caractère confidentiel des documents et de contrôler leur accessibilité. Les documents sont enfin archivés dans le système en adoptant des mesures permettant d'assurer une conservation pérenne. Ces opérations de gestion doivent se faire en conformité avec les normes et les procédures organisationnelles ainsi qu'avec l'environnement légal et réglementaire auquel l'organisation est soumise. À l'issue de ces opérations, le système de GID produit des documents bien gérés, classés et indexés avec des règles d'accès. Cela permet de favoriser leur utilisation à des fins de prise de décision et d'accomplissement des activités quotidiennes.

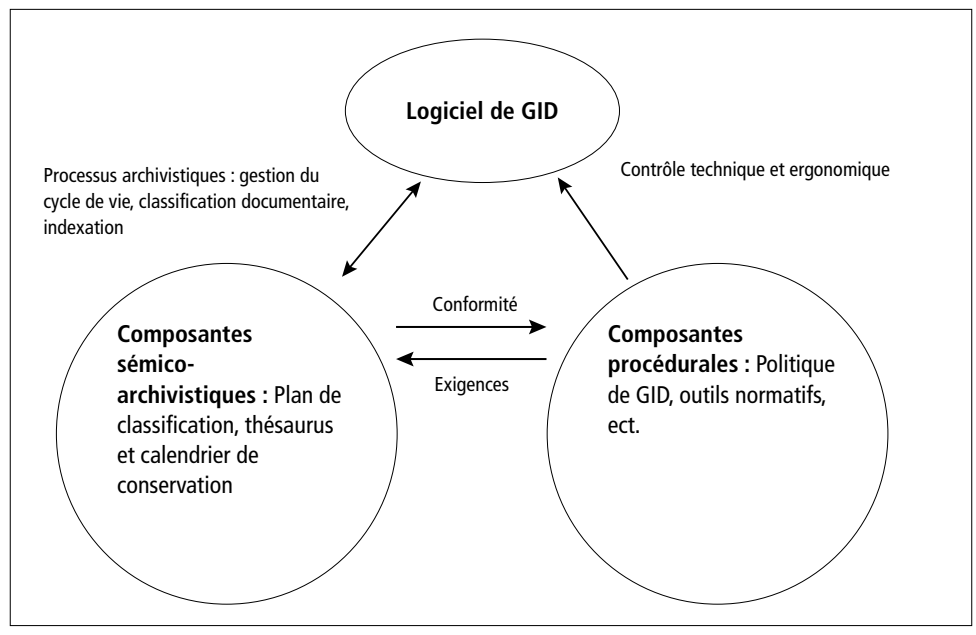

Figure 1: Composantes d'un système de GID 


\subsection{Implantation des systèmes de GID: avantages et difficultés}

La littérature professionnelle en archivistique moderne fait état des retours d'expérience en matière d'implantation des systèmes de GID. Nombreux sont les auteurs qui ont fait mention, dans leurs écrits, des caractéristiques des projets de GID réalisés au sein de leurs organisations. Pour ce qui est des avantages, Bourhis (2009-2010), Beaupré (2010-2011) et Couture (2010-2011) postulent que l'implantation des systèmes de GID a favorisé une meilleure gestion des documents dans la diversité de leurs supports: l'échange de l'information aisé entre les bases de données des documents actifs, semi-actifs et inactifs, le repérage favorisé des documents aux différentes étapes de leur cycle de vie, l'exploitabilité renforcée des documents à des fins de recherche rétrospective, I'usage maximisé des documents pour la prise de décision et la conservation sécurisée des documents historiques. Quant aux difficultés signalées, elles sont en grande partie liées, selon Couture (2010-2011), au temps considérable requis pour la réalisation des projets de GID, à la complexité des systèmes et des opérations de migration des données de l'ancien système documentaire vers le nouveau système implanté ainsi qu'à la sécurité des documents stockés dans les serveurs informatiques.

En définitive, les systèmes de GID sont des systèmes dits hybrides qui comprennent une variété d'éléments complexes en interaction qui doivent (1) être interopérables avec les autres SI (2) être alignés avec la stratégie organisationnelle et (3) offrir des fonctionnalités en adéquation avec les besoins de l'organisation, en vue d'être utilisés par l'ensemble des employés à des fins de prise de décision. Par conséquent, toute organisation doit disposer de lignes directrices ou d'un cadre normatif à suivre pour piloter l'implantation des systèmes de GID, tout en respectant les prescriptions normatives et légales régissant le champ de l'archivistique et de la gestion des documents d'activité.

\section{LA NORMALISATION: QUEL APPORT À LA GID?}

\subsection{Pourquoi normaliser?}

La normalisation est l'acte d'élaborer des lignes directrices, des recommandations ou des prescriptions spécifiques dans un objectif de régir un ensemble de pratiques relevant d'un domaine d'activité donné. 
Elle s'établit par un consensus au sein duquel de nombreux spécialistes ayant des intérêts communs se regroupent, unissent leurs efforts et partagent leur savoir-faire au profit de leur communauté. Les normes constituent le résultat de ce consensus.

La normalisation est un facteur clé d'innovation dans plusieurs champs d'activité. Elle reflète le degré de maturité des acteurs impliqués dans un domaine donné, leur conscience des enjeux et leur désir de les surmonter par l'établissement de pratiques uniformisées conduisant à un meilleur rendement et un rayonnement plus visible. De même, elle facilite la communication et l'échange des idées et pratiques entre des communautés internationales relevant du même domaine de spécialisation.

Dans le domaine de l'archivistique et de la gestion des documents d'activité, la normalisation occupe une place importante. Dupre Barnes (2011) souligne l'importance de la normalisation des pratiques de gestion des documents et considère que les normes internationales sont des moyens pour la diffusion des innovations en technologie et le partage des bonnes pratiques en matière de gestion et d'évaluation de la conformité. C'est grâce à la normalisation que le savoir-faire en matière de gestion documentaire peut être diffusé à travers le monde, puisque les méthodes de travail sont uniformisées. Bolton (2011) affirme que les normes sont un mécanisme clé pour la maîtrise des outils conçus pour l'élaboration et l'amélioration des programmes de gestion des documents. D'un point de vue technique, la réussite des projets d'implantation des systèmes de gestion documentaire nécessite la disposition d'un cadre normatif définissant les contraintes réglementaires, techniques et archivistiques auxquelles il convient de se conformer. C'est ce qui sera détaillé dans les lignes subséquentes.

\subsection{La série des normes ISO 30300 et ISO 30301 pour la mise en place d'un système de gestion des documents d'activité}

La série des normes ISO 30300: Information et documentation Systèmes de gestion des documents d'activité - Principes essentiels et vocabulaire et ISO 30301 : Information et documentation - Système de gestion des documents d'activité - Exigences a été publiée en 2011. Elle présente les principes et les exigences relatifs à la mise en place d'un 
système de gestion des documents d'activité (SGDA) dans un contexte organisationnel. Elle explique les raisons qui motivent la création d'un SGDA et les principes directeurs à suivre pour réussir sa mise en œuvre. Elle fait la liste des buts qu'un SGDA permet d'atteindre, décrit une approche axée sur le processus et détermine les rôles au sein de la direction. Cette norme peut donc être appliquée par toute organisation envisageant d'implanter un SGDA afin de soutenir ses activités (Cottin et Dessolin-Baumann, 2012).

Cette série de normes est complémentaire à la première version de la norme ISO 15489 publiée en 2001, ainsi que celle publiée en 2016 (Information et documentation - Gestion des documents d'activité) ${ }^{11}$. Celle-ci décrit les exigences que les documents d'activité doivent satisfaire pour servir de preuve et d'information pour les responsables des organisations. Elle fait la liste des marches à suivre pour la création, la capture et la gestion des documents d'activité (c'est-à-dire les opérations de classement, l'indexation, le contrôle d'accès, le stockage, I'utilisation, la migration et le sort final), et ce, en vue de concevoir et de mettre en œuvre un SGDA (ISO 15489 2016). Néanmoins, cette norme ne considère pas la gestion des documents d'activité comme processus d'affaires devant être aligné avec la stratégie globale de l'organisation. Les normes ISO 30300 et ISO 30301 définissent des principes favorisant I'alignement du processus de gestion des documents d'activité avec la stratégie organisationnelle. Elles détaillent les exigences auxquelles le SGDA doit se conformer pour qu'il soit interopérable avec les autres systèmes d'information existants dans l'organisation.

L'ISO 30300 et I'ISO 30301 fournissent des principes directeurs relatifs à l'implantation des SGDA. Ces derniers sont les produits des activités quotidiennes des organisations et sont fréquemment utilisés pour la réalisation de celles-ci. La première norme comporte des principes de base et du vocabulaire technique relatif à l'implantation des SGDA, tandis que la deuxième fournit des spécifications techniques et des exigences relatives au processus de l'implantation de ces systèmes.

\subsubsection{La norme ISO 30300}

La norme ISO 30300 (2011) se base sur des principes de gestion de qualité, visant à garantir l'alignement des SGDA avec la stratégie 
organisationnelle globale et leur interopérabilité avec les autres SI. Ces principes sont au nombre de huit (ISO 30300 2011):

- I'orientation client selon laquelle il est crucial d'assimiler les besoins des clients afin de les adapter aux produits et services offerts;

- le leadership des dirigeants, qui permet de fixer l'orientation de l'organisation;

- I'implication du personnel à tous les niveaux dans l'objectif de favoriser le succès des activités;

- I'approche par processus, qui consiste à regrouper les activités de l'organisation en processus afin d'en faciliter la réalisation et le contrôle ;

- la gestion par système afin d'identifier, de comprendre et de gérer les processus;

- I'amélioration continue des performances de l'organisation par le suivi et l'évaluation;

- I'approche factuelle pour les prises de décision, basée sur l'analyse des données et des informations;

- les relations mutuellement bénéfiques entre les fournisseurs.

Concrètement, pour que l'implantation du SGDA soit alignée avec la stratégie globale de l'organisation, elle doit être conforme à ces principes de gestion. Les attentes des clients doivent être prises en compte pour améliorer la qualité des processus de gestion des documents d'activité. La compréhension de ces besoins informationnels permet de cerner les aspects clés pour conduire l'organisation vers de meilleures performances. La direction doit exercer un rôle de leadership et de contrôle dans l'implantation du système. Elle doit élaborer une politique définissant les rôles et responsabilités des acteurs impliqués dans cette activité. Les activités incluses dans le SGDA, entre autres la création et la gestion des documents d'activité, doivent être organisées selon des processus. Cela favorise leur contrôle et leur évaluation. La gestion par système est utile dans la mesure où il permet de comprendre ces processus, de les contrôler et d'en favoriser une meilleure rentabilité. II importe que la direction assure un contrôle régulier et une évaluation continue de la performance afin d'améliorer la qualité des systèmes existants. Elle doit 
appuyer ses décisions sur des informations fiables et de qualité. Celles-ci sont fournies par des systèmes performants dotés de mesures pour assurer la fiabilité et l'intégrité du contenu. Enfin, il est crucial que l'organisation entretienne des relations bénéfiques avec les fournisseurs afin d'offrir aux clients des produits et services de qualité.

Ces mêmes principes peuvent également s'appliquer à l'implantation d'un système de GID. La connaissance des besoins des parties prenantes est cruciale dans tout projet d'implantation des SI. Le rôle de la direction est primordial dans les projets d'implantation de ces systèmes, puisque c'est à elle que la responsabilité du contrôle du déroulement du projet et de la motivation des employés incombe. Ceux-ci doivent être suffisamment motivés pour être impliqués dans le projet afin de s'investir et de contribuer efficacement à sa réalisation. L'ensemble des opérations techniques réalisées durant l'implantation du système de GID, entre autres la numérisation des documents, leur migration et l'alimentation des bases de données, doivent se réaliser dans des processus bien organisés. II importe de définir les rôles et les responsabilités de chaque intervenant dans ces processus afin de mieux contrôler ces derniers. La gestion par système est également importante dans la réalisation de ces projets. Le système de GID est, comme tous les systèmes documentaires modernes, intégré aux autres SI de l'organisation, puisque les employés se servent des documents pour l'accomplissement des activités stratégiques et opérationnelles (Margulis, 2010-2011). Ainsi, il est nécessaire de s'assurer de la performance de ces SI, de leur qualité et de leur interopérabilité avec le système de GID à établir. Après le déploiement du système, la direction doit établir des évaluations continues en vue de s'assurer de la performance du système de GID et de ses apports au rendement organisationnel. S'il est jugé performant, il servira de base pour une meilleure prise de décision, basée sur des informations fiables et authentiques. Enfin, la communication avec les fournisseurs pourrait être légitime pour bénéficier du soutien technique relatif à l'utilisation du système de GID acquis. L'application de tels principes garantira une bonne gestion de projets, en palliant ainsi les difficultés rencontrées pendant I'implantation du système de GID. C'est ainsi que la norme ISO 30300 fournit des principes directeurs qui se rapportent à la gestion de projet, partie intégrante des pratiques managériales des organisations, et qui incitent à conjuguer des considérations liées à la direction, aux employés et aux parties prenantes pour la bonne conduite des projets d'implantation des systèmes de GID. 


\subsubsection{La norme ISO 30301}

L'ISO 30301 (2011) détaille les exigences de la mise en place d'un SGDA dans un contexte organisationnel. Plus précisément, elle fait la liste des conditions qu'un SGDA doit satisfaire pour produire des informations de qualité et soutenir ainsi les processus d'affaires de l'organisation. Ces exigences sont au nombre de cinq (ISO 30301, 2011): la fiabilité, selon laquelle le système doit contenir des informations fiables qui servent de preuve aux responsables et permettent au personnel de conduire les activités quotidiennes; la sécurité qui consiste à définir des mesures dans le système afin de contrôler l'accès aux documents; la conformité qui renvoie au respect des règles et des politiques organisationnelles, de même que des exigences techniques pour assurer, dans la mesure du possible, une conservation sécurisée des documents; I'exhaustivité en vertu de laquelle le système devrait permettre d'inclure les documents reflétant les activités stratégiques de l'organisation et le caractère systématique qui réfère à l'utilisation des fonctionnalités du système par l'ensemble du personnel concerné.

Un bon SGDA est celui qui produit des informations fiables et authentiques servant de soutien à la bonne conduite des affaires organisationnelles. C'est également un système qui satisfait aux exigences en matière de sécurité (par exemple, la protection de l'information essentielle contre les actes de vol et de modifications non permises) et de stockage pérenne des documents. Un SGDA performant assure une gestion optimisée des documents décrivant les activités stratégiques de l'organisation et dont les fonctionnalités peuvent être facilement utilisées par les employés des différentes unités administratives.

La norme détaille, en sus de ces exigences, d'autres conditions dont l'organisation doit tenir compte au moment de l'implantation d'un SGDA et qui sont de nature opérationnelle. II s'agit des exigences relatives à la réalisation effective des processus d'affaires organisationnels. Elles sont liées aux performances de l'organisation, à la planification et au développement futurs, à la gestion des risques et au plan de continuité des activités (ISO 30301, 2012, section 4). En outre, I'implantation d'un SGDA doit tenir compte de l'environnement interne et externe de I'organisation (ISO 30301, 2012, section 4). Le premier comprend entre autres la gouvernance de l'organisation, la structure organisationnelle et les politiques élaborées par la direction. II inclut aussi les objectifs de 
I'organisation et les stratégies pour les atteindre, les SI existants, la forme et l'étendue des relations contractuelles de l'organisation. Le deuxième désigne l'environnement socioculturel, juridique, financier, institutionnel et technologique de l'organisation. Les relations avec les parties prenantes externes font également partie du contexte externe de l'organisation.

Un système de GID doit se conformer aux mêmes principes mentionnés plus haut. Les responsables doivent s'assurer de la fiabilité de l'information entrant dans le système (entité: intrants, voir annexe 1) de telle manière qu'elle reflète, le plus fidèlement possible, les activités de l'organisation. Ce système doit comporter des mesures permettant de vérifier l'identité des utilisateurs grâce à des mesures de sécurité afin de protéger l'information qui y est contenue. II est également impératif qu'il soit conforme non seulement aux règles de l'organisation (entité: procédures, annexe 1), mais aussi aux exigences techniques (qualité et performance du système) et celles de l'environnement externe (environnement socioculturel, institutionnel, etc.). Le système de GID doit être exhaustif pour couvrir au maximum les activités essentielles de l'organisation. En outre, s'il offre des fonctionnalités simples et faciles à utiliser, cela favorisera son utilisation systématique par l'ensemble du personnel pour soutenir la réalisation de ses activités.

La prise en compte de l'ensemble de ces considérations lors de la mise en place d'un système de GID permet de disposer d'un système fiable, performant, reflétant la réalité de l'organisation et son contexte interne et externe. C'est ainsi que toute organisation pourra maximiser l'usage de son système afin d'améliorer sa productivité, tout en se conformant aux exigences archivistiques, légales et techniques.

\subsubsection{La série des normes ISO 30300, ISO 30301 et la GID: avantages}

L'utilisation de cette série des normes représente plusieurs avantages en matière de gestion documentaire pour les organisations, entre autres, elle:

fournit un cadre pour la gouvernance des documents d'activité $[\ldots]$ et présente la méthodologie pour une approche systématique de la création et de la gestion des documents d'activité alignée sur les objectifs et stratégies de l'organisme.[Elle vise ainsi à] relier la gestion des documents d'activité au succès et à la responsabilité organisationnels (Cottin et Dessolin-Baumann, 2012, p. 129). 
En outre, elle établit des référentiels permettant aux organisations d'évaluer la qualité de leurs méthodes de gestion de documents. Elle fournit aux responsables les outils nécessaires pour assurer un contrôle systématique de la création et de la gestion des documents d'activité. Elle énumère des spécifications sur la gestion des risques. C'est ainsi que toute organisation sera de plus en plus consciente des risques découlant de la gestion et de la conservation de ses documents dans le cadre de la gouvernance d'information. Celle-ci exige l'adoption des mesures pour faire face à ces défis et assurer la sécurité des documents nécessaires à la conduite des activités stratégiques et opérationnelles. Avec des informations fiables, authentiques et intègres, les organisations seront en mesure de se défendre en cas de litige, et de faire preuve d'une bonne vigilance face aux enjeux de leurs environnements interne et externe. Il est à souligner que cette série de normes peut être utilisée avec la norme ISO 30302, qui comporte des lignes directrices « opérationnelles » sur la mise en œuvre d'un SGDA dans toute sorte d'organisation désireuse de mettre en place un système de gestion documentaire (ISO 30302, 2015).

\subsubsection{La série des normes ISO 30300, ISO 30301 et la GID: limites}

Bien qu'elle offre des avantages considérables, la série des normes ISO 30300 et ISO 30301 représente certaines limites. Comme leurs titres I'indiquent, ces normes ne concernent que les documents d'activité. Or, la GID vise à gérer l'ensemble du cycle de vie des documents, c'est-à-dire depuis leur création jusqu'à leur élimination ou leur conservation permanente. Cela nous permet de conclure que ces normes sont «partiellement » bénéfiques pour l'implantation des systèmes de GID, car elles n'abordent pas les questions de l'archivage légal et de la gestion des archives définitives. Cependant, il n'en demeure pas moins essentiel d'affirmer que les organisations pourront se servir d'une autre norme internationale régissant les activités relatives à l'archivage électronique et au stockage pérenne des documents dans les systèmes de GID. II s'agit de la norme ISO 14641-1, intitulée Archivage électronique - Partie 1 : Spécifications relatives à la conception et au fonctionnement d'un système d'informations pour la conservation d'informations électroniques. 


\subsection{La norme ISO 14641 sur l'archivage électronique}

\subsubsection{Présentation de la norme}

Cette norme a été publiée en 2012, en réponse aux défis relatifs à I'archivage probatoire de l'information numérique et aux enjeux légaux liés à la gestion et la conservation des documents sur supports papier et numérique. Avec les mutations actuelles selon lesquelles le document numérique devrait remplir les mêmes fonctions que le document papier et avoir la même valeur légale (c'est-à-dire le principe de l'équivalence fonctionnelle tel que stipulé par la Loi concernant le cadre juridique des technologies de l'information, RLRQ, chapitre (-1.1), il importe de veiller à son stockage pérenne et intègre dans les systèmes documentaires. Ceux-ci représentent des défis importants en raison de la volatilité des supports de stockage et de la sécurité des contenus archivés (par exemple, l'information essentielle).

Cette norme, basée sur la norme française NF Z42-013 sur l'archivage légal des documents numériques, fournit des spécifications techniques auxquelles les systèmes de gestion documentaire (ou d'archivage électronique) doivent répondre pour une conservation pérenne des documents numériques, tout en garantissant leur intégrité. L'ensemble des spécifications offertes par cette norme offre un référentiel sur lequel toute organisation peut se baser afin d'assurer un archivage légal, probatoire et pérenne de ses documents. Plus précisément, elle énumère les détails sur les aspects touchant le stockage pérenne des documents, les opérations de migration et celles d'archivage, ainsi que l'évaluation du système (ISO 14641, 2012). S'agissant du stockage pérenne, la norme définit les formats de fichiers recommandés pour une conservation pérenne des documents tels que le format PDF (Portable Document Format) (PDF/ A-Xa: pour la préservation de la structure logique du document, et PDF/B-Xb pour la conformité visuelle du document), le format TIFF (Tagged Image File Format) pour les images et le format $\mathrm{XML}$ pour les résultats de la numérisation (ROC : reconnaissance optique des caractères). Pour ce qui est des opérations de migration, qui portent aussi bien sur la migration des fichiers que sur la migration des supports, elles doivent se réaliser sans perte d'information. En outre, les supports documentaires doivent être aussi pérennes que possible. Les responsables des organisations doivent être conscients de la question d'obsolescence des supports informatiques, en choisissant ainsi les meilleurs supports 
de conservation des documents. Une bonne façon de le faire est de réaliser une veille technologique afin d'être à l'affût des mutations technologiques actuelles en matière de préservation des documents numériques. Quant aux opérations d'archivage, elles doivent se baser sur des principes relevant de la sécurité et de l'intégrité. Pour ce faire, il est nécessaire de vérifier la qualité (par exemple, l'intégrité) des fichiers et des documents et d'assurer leur sécurité, et ce, via des mesures appropriées telles que les empreintes numériques, les filigranes et les droits d'auteur (par exemple, l'interdiction du téléchargement). Enfin, pour l'évaluation, le système doit subir des contrôles réguliers afin de vérifier sa performance et de s'assurer qu'il est conforme aux exigences archivistiques et techniques.

Comme pour tout système d'archivage électronique, ces spécifications peuvent être également appliquées aux systèmes de GID, plus particulièrement pour leurs fonctions dédiées à la conservation pérenne des documents ayant atteint le stade semi-actif ou inactif (historique) de leur cycle de vie. Ces documents doivent être compressés dans des formats de stockage pérenne, conservés sur des supports informatiques appropriés et faire l'objet d'un contrôle rigoureux afin de s'assurer de leur intégrité et de les préserver contre les modifications non autorisées. En outre, le système de GID doit subir une évaluation périodique par les responsables, et ce, dans une optique de s'assurer de sa performance et de son apport à l'organisation.

\subsubsection{La norme ISO 14641 et la GID: avantages}

Les apports de la norme à l'archivage électronique sont indéniables. Entre autres, elle favorise un stockage pérenne des documents, et permet à l'organisation de se conformer aux exigences légales régissant l'archivage probatoire des documents. En outre, elle renforce le rôle des documents dans la défense des organisations et la bonne prise de décision et valorise la double fonction jouée par les documents (c'est-àdire la fonction d'information et de preuve). La norme ISO 14641 fournit des lignes directrices clés dont toute organisation pourrait se servir pour garantir l'archivage pérenne, légal et probatoire de ses documents. C'est ainsi que la mémoire organisationnelle sera préservée et valorisée, et que les documents serviront de soutien à la réalisation effective des activités journalières de l'organisation. 
En résumé, la figure suivante décrit le positionnement des normes ISO 30300, ISO 30301 et ISO 14641 dans un contexte de gouvernance d'information dans les organisations.

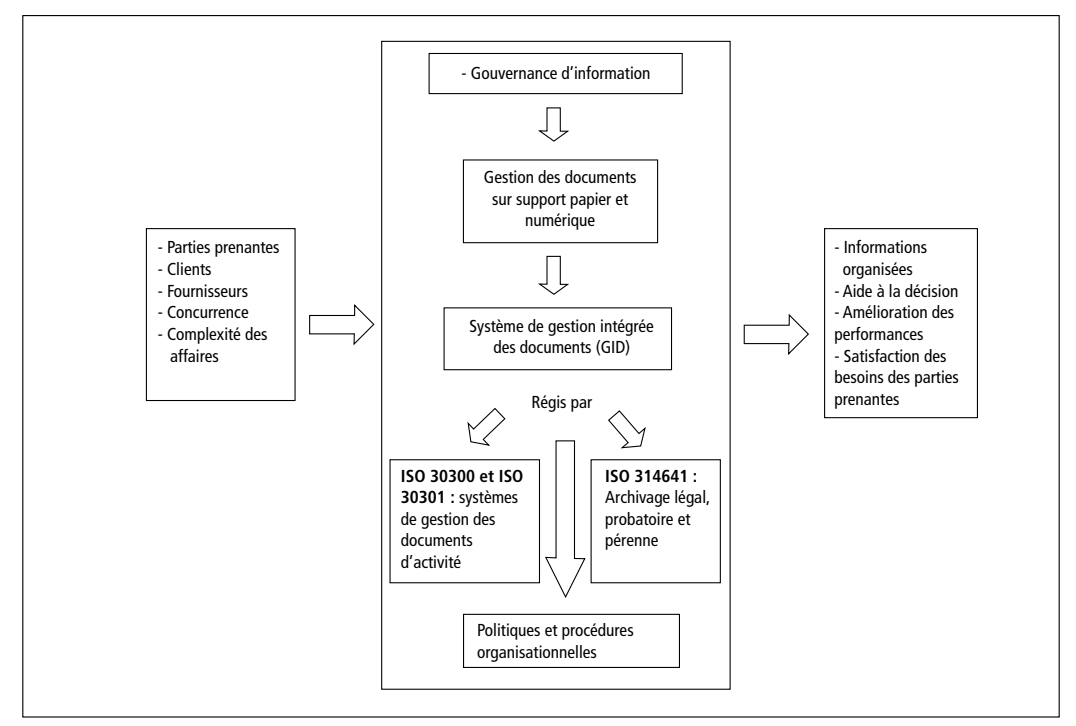

Figure 2 : Positionnement des normes ISO 30300, ISO 30301 et ISO 14641 dans la gestion documentaire

\section{CONCLUSION}

Les normes constituent un outil de pilotage et de contrôle des activités organisationnelles. Dans le cas des activités relevant de la gestion documentaire, les organisations soumises aux mêmes normes utilisent les mêmes principes et règles. Cela favorise un échange du savoir-faire entre les membres de la communauté archivistique au niveau international. La série des normes ISO 30300 et ISO 30301 ainsi que la norme ISO 14641 sont conçues pour la gestion des documents tout au long de leur cycle de vie. Les deux premières servent de soutien à la gestion des documents d'activité (c'est-à-dire les archives courantes et intermédiaires), tandis que la dernière se rapporte à l'archivage pérenne des documents numériques. Les finalités pour lesquelles ces normes ont été élaborées s'alignent avec celles de la GID, une pratique archivistique de plus en plus propagée au Québec, et qui consiste à gérer les documents sur supports papier et numérique tout au long de leur cycle de vie. Plus précisément, les normes mentionnées plus haut sont utiles pour l'implantation des systèmes de GID, 
la gestion et l'archivage sécurisé des documents. En outre, elles se basent sur des principes relevant de la norme ISO 9000 sur la qualité, qui définit les exigences de qualité auxquelles les organisations doivent se conformer afin d'offrir des produits et services répondant aux besoins de leurs clients. Ainsi, I'utilisation de ces normes permet d'assurer un contrôle de la gestion documentaire et par conséquent, de mieux répondre aux stipulations de la Loi sur les archives (RLRQ, chapitre A-21.1), incitant les organisations publiques et privées à verser une partie de leurs documents ayant une valeur historique à Bibliothèque et Archives nationales du Québec.

Ces normes peuvent être appliquées aux pratiques de gestion documentaire, tout en tenant compte de la culture organisationnelle et des stratégies des organisations désirant les utiliser. Néanmoins, force est d'admettre qu'il existe un nombre considérable de normes et de lignes directrices reconnues au niveau national qui tiennent compte de la tradition archivistique régissant chaque pays. Par exemple, au Québec, on cite le Cadre de référence gouvernemental en matière de gestion intégrée des documents (CRGGID) qui conjugue les prescriptions relatives à la gestion des documents d'activité ainsi que les documents historiques. Les spécifications qui y sont contenues sont basées, sans doute, sur les principes énoncés dans les normes internationales, notamment la norme ISO 15489, mais ayant été ajustés en fonction de la pratique archivistique québécoise. II en va de même pour la Norme pour les systèmes de gestion électronique des documents et des dossiers (SGEDD), visant entre autres à «favoriser l'efficience et l'efficacité de la gestion de l'information grâce à l'utilisation de systèmes de GEDD afin d'améliorer l'accès à une information pertinente, fiable, complète et à jour » (Gouvernement du Canada 2010). En Europe, on cite la fameuse norme MoReq (Model requirements for the management of electronic records, ou exigences types pour la maîtrise de l'archivage électronique), élaborée par la Commission européenne, et faisant la liste des spécifications techniques et fonctionnelles relatives à la gestion des documents et leur archivage pérenne dans les systèmes.

Avec la propagation de la pratique de GID à l'échelle internationale (entre autres en France et en Belgique), il devient primordial de se doter d'un cadre normatif international pour l'organisation des activités relevant de cette pratique archivistique, soit la gestion des documents sur supports papier et numérique, et ce, tout au long de leur cycle de vie. Comme pistes de recherche futures, nous suggérons que les comités techniques de la 
normalisation en archivistique déploient plus d'efforts afin d'aboutir à une réglementation normalisée de cette pratique, entre autres pour pallier, dans un premier temps, les différences liées à la terminologie utilisée. Par exemple, en France, on utilise plutôt l'appellation "gestion électronique des documents» (Guyon 2015) pour désigner aussi bien la GED que la GID. En Belgique, on commence à utiliser l'expression « gestion hybride des documents» pour parler de la même pratique (Maroye et autres, 2016). Le premier problème à pallier est donc de nature terminologique. Il reste à voir comment les pratiques seront uniformisées, tout en tenant compte de la tradition archivistique de chaque pays.

\section{Siham Alaoui}

\section{NOTES}

1. Voir le lien: http://legisquebec.gouv.qc.ca/fr/ShowDoc/cs/C-1.1 pour plus de détails sur les articles de la loi.

2. Le document est bien produit par la personne physique ou morale à laquelle il est assigné.

3. Le contenu du document n'est pas modifié ni altéré.

4. Le contenu du document reflète fidèlement les activités qu'il décrit.

5. Le document peut être communiqué et utilisé par les personnes autorisées.

6. Voir http://www.bac-lac.gc.ca/fra/a-notre-sujet/Pages/strategie-canadiennenumerisation.aspx

7. Voir http://www.banq.qc.ca/archives/archivistique gestion/ressources/publications/ gestion integree/crggid/

8. Pour plus d'informations, voir le lien http://www.ica.org/sites/default/files/Digital\%20 Preservation\%20lnitatives\%20Module 0.pdf

9. Voir le lien: http://legisquebec.gouv.qc.ca/fr/ShowDoc/cs/A-21.1

10. Voir le lien: http://legisquebec.gouv.qc.ca/fr/ShowDoc/cs/A-2.1

11. La norme constitue une version révisée de la norme ISO 15489 - Records management, publiée en 2001. Elle remplace l'appellation Records management par gestion des documents d'activité. Celle-ci était adoptée pour la première fois lors de la publication des séries des normes ISO 30300 et ISO 30301 (2011). 


\section{BIBLIOGRAPHIE}

BEAUPRÉ, L. (2010-2011). La mémoire corporative de la ville de Laval: d'hier à demain! Archives 42(1), 7-22. Repéré à https://www. archivistes.qc.ca/revuearchives/vol42 1/42 1 beaupre.pdf

BOLTON, J. (2011). Standards: providing a framework for RIM success. Information management (May-June), 30-35.

BOURHIS, T. (2009-2010). Le projet Hippocampe: la mise en place d'un système de gestion électronique des documents à I'Université de Montréal. Archives 41(2), 135-161. Repéré à https://www.archivistes. qc.ca/revuearchives/vol41 2/41 2 bourhis.pdf

CHEVRIER, J. (2010-2011). La gestion de documents à l'arrondissement de Saint-Laurent. Archives 42(1), 23-44. Repéré à: https://www.archivistes. qc.ca/revuearchives/vol42 1/42 1 chevrier.pdf

COTTIN, M. et DESSOLIN-BAUMANN, S. (2012). La famille des normes ISO sur le records management. La Gazette des archives (228), 119-133.

COUTURE, C. (2010-2011). La gestion des archives de la Ville de Brossard. Archives 42(1), 45-66. Repéré à https://www.archivistes. qc.ca/revuearchives/vol42_1/42_1_couture.pdf

DUPRE BARNES, N. (2011). Going with RIM standards. Information management (May-June), 20-24.

GOUVERNEMENT DU CANADA. (2010). Norme pour les systèmes de gestion électronique des documents et des dossiers. Repéré à: http://www.tbs-sct.gc.ca/pol/doc-fra.aspx?id=18910

GOUVERNEMENT DU QUÉBEC. (2001). La loi concernant le cadre juridique des technologies de l'information. Repéré à http://legisquebec.gouv. qc.ca/fr/ShowDoc/cs/C-1.1

GUYON, C. (2015). La pratique archivistique publique en France, entre adaptation et négociation. Les cahiers du numérique 11(2), 77-114. Repéré à https://www.cairn.info/revue-les-cahiers-du-numerique-20152-page-77.htm 
HECTOR PROJECT. (2016). Hector: Hybrid electronic curation, transformation and organization of records. Repéré à http://www. hector-project.be/?lang=fr (lien non fonctionnel)

LESAUX, A. (1992). La gestion électronique de documents. Bulletin des bibliothèques de France (4). Repéré à http://bbf.enssib.fr/consulter/ bbf-1992-04-0073-006

MARGULIS, A. (2009-2010). Identification des facteurs de succès d'implantation des SI en gestion documentaire moderne: une étude empirique. Archives 41(2), 45-78. Repéré à http://www.archivistes. qc.ca/revuearchives/vol41 2/41 2 margulis.pdf

MAROYE, L., ARANGUREN, F., DEMOULIN, M., DE TERWANGNE, C., LOSDYCK, B., SOYEZ, S., VAN HOOLAND, S. et VANRECK, O. (2016). La gestion hybride des documents au sein des administrations fédérales belges sous la loupe du projet de recherche «Hector». Pyramides 26/27, 215-230. Repéré à : https://pyramides.revues.org/1005\#text

MAS, S. (2007). Schémas de classification et repérage des documents administratifs électroniques dans un contexte de gestion décentralisée des ressources informationnelles. (Thèse de doctorat, Université de Montréal). Repéré à https://papyrus.bib.umontreal.ca/xmlui/ bitstream/handle/1866/1433/mas-s-these-schema-reperage. pdf? sequence $=1$ \&isAllowed $=y$

O'BRIEN, J. (2003). Introduction aux systèmes d'information: Un outil essentiel pour l'entreprise branchée. Montréal, Québec: Chenelière/ McGraw-Hill.

ORGANISATION INTERNATIONALE DE NORMALISATION, COMITÉ TECHNIQUE ISO.TC 46, INFORMATION ET DOCUMENTATION. (2011). ISO 30300, Information et documentation - Systèmes de gestion des documents d'activité - Principes essentiels et vocabulaire. Geneva, Suisse: ISO.

ORGANISATION INTERNATIONALE DE NORMALISATION, COMITÉ TECHNIQUE ISO.TC 46, INFORMATION ET DOCUMENTATION. (2011). ISO 30301, Information et documentation - Système de gestion des documents d'activité - Exigences. Geneva, Suisse: ISO. 
ORGANISATION INTERNATIONALE DE NORMALISATION, COMITÉ TECHNIQUE TC 171, APPLICATIONS EN GESTION DES DOCUMENTS. (2012). Archivage électronique - Partie 1: Spécifications relatives à la conception et au fonctionnement d'un système d'informations pour la conservation d'informations électroniques. Geneva, Suisse: ISO.

ORGANISATION INTERNATIONALE DE NORMALISATION, COMITÉ TECHNIQUE ISO.TC 46, INFORMATION ET DOCUMENTATION. (2015). ISO 30302, Information et documentation - Système de gestion des documents d'activité - Lignes directrices de mise en œuvre. Geneva, Suisse: ISO.

ORGANISATION INTERNATIONALE DE NORMALISATION, COMITÉ TECHNIQUE ISO.TC 46, INFORMATION ET DOCUMENTATION. (2016). ISO 15489, Information et documentation - Gestion des documents d'activité, partie 1 : concepts et principes. Geneva, Suisse: ISO.

ROBERGE, M. (2009). La gestion intégrée des documents en format papier et technologiques. Québec, Québec: Éditions Michel Roberge.

ROBERGE, M. (2016). La gestion intégrée des documents d'activité technologiques et en format papier. Québec, Québec: Éditions Michel Roberge.

UNIVERSITÉDU QUÉBEC ÀMONTRÉAL, SERVICEDES ARCHIVESETDE GESTION DES DOCUMENTS. (s.d.). Gestion intégrée des documents (GID). Repéré à: https://archives.uqam.ca/gestion-documents-numeriques/presentationprogramme.html 